\title{
KARAKTERISTIK MATRIKS CENTRO-SIMETRIS
}

\author{
Berny Pebo Tomasouw \\ Jurusan Matematika FMIPA Universitas Pattimura \\ Jl. Ir. M. Putuhena, Kampus Unpatti, Poka-Ambon, Indonesia \\ e-mail: peboberny@gmail.com
}

\begin{abstract}
Abstrak
Matriks centro-simetris merupakan matriks simetris yang memenuhi sifat tertentu. Dalam penelitian ini, akan dibahas bentuk dan beberapa sifat dasar dari matriks centro-simetris serta nilai eigen dan vektor eigennya.
\end{abstract}

Kata Kunci: Matriks centro-simetris, nilai eigen, vector eigen.

\section{THE CHARACTERISTICS OF CENTROSYMMETRIC MATRICES}

\begin{abstract}
Centrosymmetric is a symmetric matrix which satisfy some certain conditions. In this paper, we will discuss the form and some basic properties of the centrosymmetric matrix also its eigenvalues and eigenvectors.
\end{abstract}

Keywords: Centrosymmetric matrix, eigen value, eigen vector.

\section{Pendahuluan}

Konsep matriks centro-simetris muncul dalam buku yang ditulis A. C. Aitken [1] dengan judul "Determinants and Matrices". Sedangkan F. Graybill juga membahas konsep matriks yang sama namun dengan nama matriks cross-simetris [2]. Walaupun memiliki nama yang mirip dengan matriks simetris namun sifat yang dimiliki oleh matriks contro-simetris sangatlah berbeda.

Beberapa penelitian memperlihatkan bawha matriks centro-simetris mempunyai peranan penting dalam masalah analisis numerik, teori informasi, proses Markov, persamaan diferensial, persegi ajaib maupun pengenalan pola [3-8]. Oleh karena itu, dalam penelitian ini akan dibahas karakteristik dari matriks centro-simetris beserta nilai eigen dan vektor eigennya.

\section{Definisi 1. (Matriks Simetris)}

Matriks $A \in \mathbf{M}_{n}(\square)$ dikatakan simetris jika berlaku

$$
A=A^{T}
$$

\section{Definisi 2. (Nilai Eigen dan Vektor Eigen)}

Diberikan matriks $A \in \mathbf{M}_{n}(\square)$ dan vektor tak nol $\mathbf{x} \in \square^{n}$. Skalar $\lambda \in \square$ merupakan nilai eigen dari A jika memenuhi $A \mathbf{x}=\lambda \mathbf{x}$. Vektor $\mathbf{x}$ disebut vektor eigen yang bersesuaian dengan nilai eigen $\lambda$. 


\section{Definisi 3. (Matriks Similar)}

Diberikan matriks $A, B \in \mathbf{M}_{n}(\square)$. Matriks $A$ dikatakan similar dengan $B$ jika terdapat matriks non-singular $P$ sedemikian sehingga berlaku

$$
A=P B P^{-1} .
$$

Lema 1. Jika matriks $A$ similar dengan matriks $B$ maka $\operatorname{det}(A)=\operatorname{det}(B)$.

Akibat 1. Misalkan matriks $A$ similar dengan matriks $B$. Jika $\lambda$ adalah nilai eigen dari $A$ maka $\lambda$ juga merupakan nilai eigen dari $B$.

\section{Definisi 4. (Matriks Ortogonal)}

Matriks non-singular $Q \in \mathbf{M}_{n}(\square)$ disebut matriks ortogonal jika memenuhi $Q^{T}=Q^{-1}$.

\section{Definisi 5. (Matriks contra-identitas)}

Matriks persegi $J_{n}$ disebut matriks contra-identitas jika berbentuk

$$
J_{n}=\left[\begin{array}{ccccc}
0 & 0 & \cdots & 0 & 1 \\
0 & 0 & . & 1 & 0 \\
\vdots & . & . & . & \vdots \\
0 & 1 & . & 0 & 0 \\
1 & 0 & \cdots & 0 & 0
\end{array}\right]
$$

Lema 2. Matriks contra-identitas memenuhi sifat-sifat berikut :
a. $J_{n}=J_{n}^{T}$.
b. $J_{n}^{-1}=J_{n}$.
c. $J_{n}^{k}= \begin{cases}I_{n} & , \text { jika } k \text { genap } \\ J_{n} & , \text { jika } k \text { ganjil }\end{cases}$

\section{Definsi 6. (Rotasi Matriks)}

Diberikan $A \in \mathbf{M}_{n}(\square)$. Rotasi dari matriks $A$ dinotasikan dengan $A^{R}$ dan didefinisikan sebagai

$$
A^{R}=J_{n} A J_{n}
$$

Lema 3. Rotasi matriks $A$ memenuhi sifat-sifat berikut :
a. $\left(A^{R}\right)^{R}=A$.
b. $\left(A^{R}\right)^{T}=\left(A^{T}\right)^{R}$.
c. $\left(A^{-1}\right)^{R}=\left(A^{R}\right)^{-1}$, jika $A$ non-singular. 


\section{Hasil dan Pembahasan}

\section{Definisi 7. (Matriks Centro-Simetris)}

Diberikan matriks $S$ berorde $n \times n$. Matriks $S$ disebut matriks centro-simetris jika memenuhi

$$
S^{R}=S
$$

Contoh 1. Matriks $S=\left[\begin{array}{cc}3 & -2 \\ -2 & 3\end{array}\right]$ adalah matriks centro-simetris karena

$$
\begin{aligned}
S^{R} & =J_{2} S J_{2} \\
& =\left[\begin{array}{ll}
0 & 1 \\
1 & 0
\end{array}\right]\left[\begin{array}{cc}
3 & -2 \\
-2 & 3
\end{array}\right]\left[\begin{array}{ll}
0 & 1 \\
1 & 0
\end{array}\right] \\
& =\left[\begin{array}{cc}
3 & -2 \\
-2 & 3
\end{array}\right] \\
& =S
\end{aligned}
$$

Contoh 2. Matriks $S=\left[\begin{array}{lll}2 & 1 & 3 \\ 0 & 5 & 0 \\ 3 & 1 & 2\end{array}\right]$ adalah matriks centro-simetris.

Contoh $1 \mathrm{di}$ atas memperlihatkan contoh matriks simetris yang juga merupakan matriks centro-simetris. Namun hal ini tidak berlaku umum ataupun sebaliknya seperti tampak pada contoh 2 di atas. Lema 4 berikut ini memperlihatkan bentuk umum dari matriks centro-simetris berukuran $2 \times 2$ dan $3 \times 3$.

\section{Lema 4.}

i. Jika $S=\left[\begin{array}{ll}a & b \\ b & a\end{array}\right]$ maka $S$ adalah matriks centro-simetris.

ii. Jika $S=\left[\begin{array}{ccc}a & c & b \\ d & e & d \\ b & c & a\end{array}\right]$ maka $S$ adalah matriks centro-simetris.

Selanjutnya, bentuk umum matriks centro-simetris dengan orde $n \geq 4$ dibagi ke dalam 2 kasus yakni $n$ genap dan $n$ ganjil. Untuk kasus $n$ genap yakni $n=2 m$ maka misalkan matriks $S$ berbentuk matriks blok sebagai berikut

$$
S=\left[\begin{array}{ll}
A & C \\
B & D
\end{array}\right]
$$

dengan $A, B, C$, dan $D$ adalah matriks persegi berorde $m$. Matriks $S$ harus memenuhi $S^{R}=S$ sehingga

$$
\begin{aligned}
& J_{n} S J_{n}=S \\
& {\left[\begin{array}{ll}
O_{m} & J_{m} \\
J_{m} & O_{m}
\end{array}\right]\left[\begin{array}{ll}
A & C \\
B & D
\end{array}\right]\left[\begin{array}{ll}
O_{m} & J_{m} \\
J_{m} & O_{m}
\end{array}\right]=\left[\begin{array}{cc}
A & C \\
B & D
\end{array}\right]} \\
& {\left[\begin{array}{ll}
J_{m} D J_{m} & J_{m} B J_{m} \\
J_{m} C J_{m} & J_{m} A J_{m}
\end{array}\right]=\left[\begin{array}{ll}
A & C \\
B & D
\end{array}\right]}
\end{aligned}
$$

Dari bentuk terakhir diperoleh $D=J_{m} A J_{m}$ dan $C=J_{m} B J_{m}$. Hasil ini diperlihatkan dalam Lema berikut. 
Lema 5. Jika $S$ adalah matriks centro-simetris berorde n genap yakni $n=2 m$ maka $S$ dapat ditulis dalam bentruk matriks blok sebagai berikut

$$
S=\left[\begin{array}{ll}
A & J_{m} B J_{m} \\
B & J_{m} A J_{m}
\end{array}\right],
$$

dengan $A$ dan $B$ adalah matriks persegi berorde $m$.

Bentuk matriks $S$ pada Lema di atas bukanlah bentuk tunggal karena matriks $S$ dapat juga ditulis dalam bentuk

$$
S=\left[\begin{array}{cc}
A & B \\
J_{m} B J_{m} & J_{m} A J_{m}
\end{array}\right] \text { atau } S=\left[\begin{array}{cc}
A & J_{m} B \\
B J_{m} & J_{m} A J_{m}
\end{array}\right] .
$$

Contoh 3. Matriks $S=\left[\begin{array}{cccc}2 & 1 & 2 & -3 \\ 3 & 5 & 3 & 1 \\ 1 & 3 & 5 & 3 \\ -3 & 2 & 1 & 2\end{array}\right]$ adalah matriks centro-simetris karena $S$ dapat ditulis dalam bentuk $S=\left[\begin{array}{ll}A & J_{m} B J_{m} \\ B & J_{m} A J_{m}\end{array}\right]$ dengan $A=\left[\begin{array}{ll}2 & 1 \\ 3 & 5\end{array}\right]$ dan $B=\left[\begin{array}{cc}1 & 3 \\ -3 & 2\end{array}\right]$

Selanjutya, untuk matriks persegi berorde $n$ ganjil yakni $n=2 m+1$ maka misalkan $S$ berbentuk matriks blok sebagai berikut

$$
S=\left[\begin{array}{ccc}
A & \mathbf{p} & C \\
\mathbf{q}^{T} & \alpha & \mathbf{r}^{T} \\
B & \mathbf{s} & D
\end{array}\right]
$$

dengan $A, B, C, D$ adalah matriks persegi berorde $m$, vektor $\mathbf{p}, \mathbf{q}, \mathbf{r}, \mathbf{s} \in \square^{m}$ dan $\alpha \in \square$. Matriks $S$ harus memenuhi $S^{R}=S$ sehingga

$$
\begin{gathered}
J_{n} S J_{n}=S \\
{\left[\begin{array}{lll}
O_{m} & \mathbf{0} & J_{m} \\
\mathbf{0}^{T} & 1 & \mathbf{0}^{T} \\
J_{m} & \mathbf{0} & O_{m}
\end{array}\right]\left[\begin{array}{ccc}
A & \mathbf{p} & C \\
\mathbf{q}^{T} & \alpha & \mathbf{r}^{T} \\
B & \mathbf{s} & D
\end{array}\right]\left[\begin{array}{ccc}
O_{m} & \mathbf{0} & J_{m} \\
\mathbf{0}^{T} & 1 & \mathbf{0}^{T} \\
J_{m} & \mathbf{0} & O_{m}
\end{array}\right]=\left[\begin{array}{ccc}
A & \mathbf{p} & C \\
\mathbf{q}^{T} & \alpha & \mathbf{r}^{T} \\
B & \mathbf{s} & D
\end{array}\right]} \\
{\left[\begin{array}{ccc}
J_{m} D J_{m} & J_{m} \mathbf{s} & J_{m} B J_{m} \\
\mathbf{r}^{T} J_{m} & \alpha & \mathbf{q}^{T} J_{m} \\
J_{m} C J_{m} & J_{m} \mathbf{p} & J_{m} A J_{m}
\end{array}\right]=\left[\begin{array}{ccc}
A & \mathbf{p} & C \\
\mathbf{q}^{T} & \alpha & \mathbf{r}^{T} \\
B & \mathbf{s} & D
\end{array}\right]}
\end{gathered}
$$

Dari bentuk terakhir diperoleh persamaan :

i. $\quad J_{m} D J_{m}=A$ dan $D=J_{m} A J_{m}$.

ii. $J_{m} \mathbf{s}=\mathbf{p}$ dan $J_{m} \mathbf{p}=\mathbf{s}$.

iii. $\mathbf{r}^{T} J_{m}=\mathbf{q}^{T}$ dan $\mathbf{r}^{T}=\mathbf{q}^{T} J_{m}$.

iv. $J_{m} C J_{m}=B$ dan $C=J_{m} B J_{m}$.

Dari hasil ini dapat dibentuk Lema berikut. 
Lema 6. Jika $S$ adalah matriks centro-simetris berorde $n$ ganjil yakni $n=2 m+1$ maka $S$ dapat ditulis dalam bentruk matriks blok sebagai berikut

$$
S=\left[\begin{array}{ccc}
A & \mathbf{p} & J_{m} B J_{m} \\
\mathbf{q}^{T} & \alpha & \mathbf{q}^{T} J_{m} \\
B & J_{m} \mathbf{p} & J_{m} A J_{m}
\end{array}\right],
$$

dengan $A, B$ adalah matriks persegi berorde $m$, vektor $\mathbf{p}, \mathbf{q} \in \square^{m}$ dan $\alpha \in \square$.

Selanjutnya, Lema berikut akan membantu dalam mencari matriks yang similar dengan matriks centrosimetris.

\section{Lema 7.}

a. Matriks $Q_{1}=\frac{1}{\sqrt{2}}\left[\begin{array}{cc}I_{m} & I_{m} \\ -J_{m} & J_{m}\end{array}\right]$ adalah matriks ortogonal berorde $n=2 m$.

b. Matriks $Q_{2}=\frac{1}{\sqrt{2}}\left[\begin{array}{ccc}I_{m} & \mathbf{0} & I_{m} \\ \mathbf{0}^{T} & \sqrt{2} & \mathbf{0}^{T} \\ -J_{m} & \mathbf{0} & J_{m}\end{array}\right]$ adalah matriks ortogonal berorde $n=2 m+1$.

Dengan menggunakan matriks $Q_{1}$ dan $Q_{2}$ pada Lema ini maka dapat dicari matriks yang similar dengan matriks centro-simetris.

Untuk kasus $n$ genap maka misalkan $D_{1}$ adalah matriks yang similar. Matriks centro-simetris $S$ dan $D_{1}$ harus memenuhi $S=Q_{1} D_{1} Q_{1}^{T}$ atau

$$
\begin{aligned}
D_{1} & =Q_{1}^{T} S Q_{1} \\
& =\left(\frac{1}{\sqrt{2}}\left[\begin{array}{cc}
I_{m} & -J_{m} \\
I_{m} & J_{m}
\end{array}\right]\right)\left[\begin{array}{cc}
A & J_{m} B J_{m} \\
B & J_{m} A J_{m}
\end{array}\right]\left(\frac{1}{\sqrt{2}}\left[\begin{array}{cc}
I_{m} & I_{m} \\
-J_{m} & J_{m}
\end{array}\right]\right) \\
& =\frac{1}{2}\left[\begin{array}{cc}
2\left(A-J_{m} B\right) & O_{m} \\
O_{m} & 2\left(A+J_{m} B\right)
\end{array}\right] \\
& =\left[\begin{array}{cc}
A-J_{m} B & O_{m} \\
O_{m} & A+J_{m} B
\end{array}\right]
\end{aligned}
$$

Sedangkan untuk kasus n ganjil diperoleh matriks $D_{2}$ yang similar yaitu

$$
\begin{aligned}
D_{2} & =Q_{2}^{T} S Q_{2} \\
& =\left(\frac{1}{\sqrt{2}}\left[\begin{array}{ccc}
I_{m} & \mathbf{0} & -J_{m} \\
\mathbf{0}^{T} & \sqrt{2} & \mathbf{0}^{T} \\
I_{m} & \mathbf{0} & J_{m}
\end{array}\right]\right)\left[\begin{array}{ccc}
A & \mathbf{p} & J_{m} B J_{m} \\
\mathbf{q}^{T} & \alpha & \mathbf{q}^{T} J_{m} \\
B & J_{m} \mathbf{p} & J_{m} A J_{m}
\end{array}\right]\left(\frac{1}{\sqrt{2}}\left[\begin{array}{ccc}
I_{m} & \mathbf{0} & I_{m} \\
\mathbf{0}^{T} & \sqrt{2} & \mathbf{0}^{T} \\
-J_{m} & \mathbf{0} & J_{m}
\end{array}\right]\right) \\
& =\frac{1}{2}\left[\begin{array}{ccc}
2\left(A-J_{m} B\right) & \mathbf{0} & O_{m} \\
\mathbf{0}^{T} & 2 \alpha & 2 \sqrt{2} \mathbf{q}^{T} \\
O_{m} & 2 \sqrt{2} \mathbf{p} & 2\left(A+J_{m} B\right)
\end{array}\right]
\end{aligned}
$$




$$
=\left[\begin{array}{ccc}
A-J_{m} B & \mathbf{0} & O_{m} \\
\mathbf{0}^{T} & \alpha & \sqrt{2} \mathbf{q}^{T} \\
O_{m} & \sqrt{2} \mathbf{p} & A+J_{m} B
\end{array}\right]
$$

Dari hasil ini dapat dibentuk Teorema berikut.

\section{Teorema 1.}

a. Jika $S=\left[\begin{array}{cc}A & J_{m} B J_{m} \\ B & J_{m} A J_{m}\end{array}\right]$ dan $Q_{1}=\frac{1}{\sqrt{2}}\left[\begin{array}{cc}I_{m} & I_{m} \\ -J_{m} & J_{m}\end{array}\right]$ maka $S$ similar dengan matriks diagonal blok $D_{1}=\left[\begin{array}{cc}A-J_{m} B & O_{m} \\ O_{m} & A+J_{m} B\end{array}\right]$.

b. Jika $S=\left[\begin{array}{ccc}A & \mathbf{p} & J_{m} B J_{m} \\ \mathbf{q}^{T} & \alpha & \mathbf{q}^{T} J_{m} \\ B & J_{m} \mathbf{p} & J_{m} A J_{m}\end{array}\right]$ dan $Q_{2}=\frac{1}{\sqrt{2}}\left[\begin{array}{ccc}I_{m} & \mathbf{0} & I_{m} \\ \mathbf{0}^{T} & \sqrt{2} & \mathbf{0}^{T} \\ -J_{m} & \mathbf{0} & J_{m}\end{array}\right]$ maka $S$ similar dengan matriks diagonal blok $D_{2}=\left[\begin{array}{ccc}A-J_{m} B & \mathbf{0} & O_{m} \\ \mathbf{0}^{T} & \alpha & \sqrt{2} \mathbf{q}^{T} \\ O_{m} & \sqrt{2} \mathbf{p} & A+J_{m} B\end{array}\right]$

Dari Teorema 1 diperoleh tiga akibat sebagai berikut.

Akibat 2. Determinan dari matriks centro-simetris adalah

a. Untuk $n$ genap

$$
\operatorname{det}\left(\left[\begin{array}{ll}
A & J_{m} B J_{m} \\
B & J_{m} A J_{m}
\end{array}\right]\right)=\operatorname{det}\left(A-J_{m} B\right) \operatorname{det}\left(A+J_{m} B\right)
$$

b. Untuk $n$ ganjil

$$
\operatorname{det}\left(\left[\begin{array}{ccc}
A & \mathbf{p} & J_{m} B J_{m} \\
\mathbf{q}^{T} & \alpha & \mathbf{q}^{T} J_{m} \\
B & J_{m} \mathbf{p} & J_{m} A J_{m}
\end{array}\right]\right)=\operatorname{det}\left(A-J_{m} B\right) \operatorname{det}\left(\left[\begin{array}{cc}
\alpha & \sqrt{2} \mathbf{q}^{T} \\
\sqrt{2} \mathbf{p} & A+J_{m} B
\end{array}\right]\right) .
$$

Akibat 3. Jika $S=\left[\begin{array}{cc}A & J_{m} B J_{m} \\ B & J_{m} A J_{m}\end{array}\right]$ serta matriks $A-J_{m} B$ dan $A+J_{m} B$ tak singular maka

$$
S^{-1}=\frac{1}{2}\left[\begin{array}{cc}
F^{-1}+G^{-1} & \left(G^{-1}-F^{-1}\right) J_{m} \\
J_{m}\left(G^{-1}-F^{-1}\right) & J_{m}\left(F^{-1}+G^{-1}\right) J_{m}
\end{array}\right] \text {. }
$$

dengan $F=A-J_{m} B$ dan $G=A+J_{m} B$. 
Akibat 4. Nilai eigen dari matriks centro-simetris adalah

a. Untuk $n$ genap

Nilai eigen dari $S=\left[\begin{array}{cc}A & J_{m} B J_{m} \\ B & J_{m} A J_{m}\end{array}\right]$ adalah nilai eigen dari matriks $A-J_{m} B$ dan nilai eigen dari matriks $A+J_{m} B$.

b. Untuk $n$ ganjil

Nilai eigen dari $S=\left[\begin{array}{ccc}A & \mathbf{p} & J_{m} B J_{m} \\ \mathbf{q}^{T} & \alpha & \mathbf{q}^{T} J_{m} \\ B & J_{m} \mathbf{p} & J_{m} A J_{m}\end{array}\right]$ adalah nilai eigen dari matriks $A-J_{m} B$ dan nilai eigen dari matriks $\left[\begin{array}{cc}\alpha & \sqrt{2} \mathbf{q}^{T} \\ \sqrt{2} \mathbf{p} & A+J_{m} B\end{array}\right]$

Bentuk vektor eigen dari matriks centro-simetris diperlihatkan dalam Teorema berikut.

Teorema 2. Diberikan matriks centro-simetris $S=\left[\begin{array}{cc}A & J_{m} B J_{m} \\ B & J_{m} A J_{m}\end{array}\right]$.

a. Jika vektor eigen dari $A-J_{m} B$ adalah $\mathbf{y}$ maka vektor eigen dari $S$ adalah $\mathbf{v}=\left[\begin{array}{c}\mathbf{y} \\ -J_{m} \mathbf{y}\end{array}\right]$.

b. Jika vektor eigen dari $A+J_{m} B$ adalah $\mathbf{z}$ maka vektor eigen dari $S$ adalah $\mathbf{w}=\left[\begin{array}{c}\mathbf{z} \\ J_{m} \mathbf{z}\end{array}\right]$.

\section{Bukti.}

a. Misalkan nilai eigen dari $A-J_{m} B$ adalah $\lambda$ dengan vektor eigen yang bersesuaian adalah $\mathbf{y}$. Harus ditunjukkan bahwa berlaku $S \mathbf{v}=\lambda \mathbf{v}$.

$$
\begin{aligned}
S \mathbf{v} & =\left[\begin{array}{ll}
A & J_{m} B J_{m} \\
B & J_{m} A J_{m}
\end{array}\right]\left[\begin{array}{c}
\mathbf{y} \\
-J_{m} \mathbf{y}
\end{array}\right] \\
& =\left[\begin{array}{l}
A \mathbf{y}-J_{m} B \mathbf{y} \\
B \mathbf{y}-J_{m} A \mathbf{y}
\end{array}\right] \\
& =\left[\begin{array}{l}
\left(A-J_{m} B\right) \mathbf{y} \\
-J_{m}\left(A-J_{m} B\right) \mathbf{y}
\end{array}\right] \\
& =\left[\begin{array}{l}
\lambda \mathbf{y} \\
-J_{m} \lambda \mathbf{y}
\end{array}\right] \\
& =\lambda\left[\begin{array}{l}
\mathbf{y} \\
-J_{m} \mathbf{y}
\end{array}\right] \\
& =\lambda \mathbf{v}
\end{aligned}
$$

Karena $S \mathbf{v}=\lambda \mathbf{v}$ maka terbukti $\mathbf{v}=\left[\begin{array}{c}\mathbf{y} \\ -J_{m} \mathbf{y}\end{array}\right]$ adalah vektor eigen dari $S$. 
b. Misalkan nilai eigen dari $A+J_{m} B$ adalah $\beta$ dengan vektor eigen yang bersesuaian adalah $\mathbf{z}$. Harus ditunjukkan bahwa berlaku $S \mathbf{w}=\beta \mathbf{w}$.

$$
\begin{aligned}
S \mathbf{v} & =\left[\begin{array}{ll}
A & J_{m} B J_{m} \\
B & J_{m} A J_{m}
\end{array}\right]\left[\begin{array}{c}
\mathbf{z} \\
J_{m} \mathbf{z}
\end{array}\right] \\
& =\left[\begin{array}{l}
A \mathbf{z}+J_{m} B \mathbf{z} \\
B \mathbf{z}+J_{m} A \mathbf{z}
\end{array}\right] \\
& =\left[\begin{array}{l}
\left(A+J_{m} B\right) \mathbf{z} \\
J_{m}\left(A+J_{m} B\right) \mathbf{z}
\end{array}\right] \\
& =\left[\begin{array}{l}
\beta \mathbf{z} \\
J_{m} \beta \mathbf{z}
\end{array}\right] \\
& =\beta\left[\begin{array}{c}
\mathbf{z} \\
J_{m} \mathbf{z}
\end{array}\right] \\
& =\beta \mathbf{w}
\end{aligned}
$$

Karena $S \mathbf{w}=\beta \mathbf{w}$ maka terbukti bahwa $\mathbf{w}=\left[\begin{array}{c}\mathbf{z} \\ J_{m} \mathbf{z}\end{array}\right]$ adalah vektor eigen dari $S$.

\section{Daftar Pustaka}

[1] A.C.Aitken., Determinants and Matrices, Edinburgh. Oliver and Boyd, 1939.

[2] F. Graybill, Introduction to Matrices with Application in Statistics, Wadsworth. Belmont., 1969.

[3] A. C. a. P. Butler, "Properties of the Eigen vectors of Persymmetric Matrices with Applications to Communication Theory. IEEE Transactions on Communications.," vol. 24, pp. pp 804-809, 1976.

[4] J. Delmas, "On Adaptive EVD Asymptotic Distribution of Centro-Symmetric Covariance Matrices". IEEE Transactions on Signal Processing," Vol.47, pp.1402-1406, 1999.

[5] W. Chen, Y.Yu and X. Wang, "Reducing the Computational Requirement of Differential Quadrature Method". Numerical Methods for Partial Differential Equations," Vol.12, pp.565-577, 1996.

[6] F. Stenger, "Matrices of Sinc Methods," Journal of Computational and Applied Mathematics, Vol. 86, pp.297-310, 1997.

[7] R. B. Mattingly, "Even Order Regular Magic Squares Are Singular," The American Mathematical Monthly, Vol.107, pp. pp.777-782, 2000.

[8] L. Datta and S. Morgera, "Some Results on Matrix Symmetries and a Pattern Recognition Application," IEEE Transactions on Signal Processing, Vol.34, No.4, pp.992-994, 1986. 